\title{
Serum levels of advanced glycation end products in type II diabetic patients with acute cerebral infarction.
}

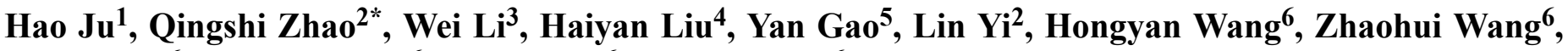 \\ Chuan Liu', Fanhua Meng ${ }^{6}$, Haiping Li ${ }^{6}$, Ligang Jiang6 \\ ${ }^{1}$ Department of Endocrinology, Affiliated Hospital of Beihua University, Jilin, PR China \\ ${ }^{2}$ Stroke Ward, Affiliated Hospital of Beihua University, Jilin, PR China \\ ${ }^{3}$ Department of Neurology, Affiliated Hospital of Beihua University, Jilin, PR China \\ ${ }^{4}$ Department of Neurology, Jilin people's Hospital, Jilin, PR China \\ ${ }^{5}$ Stroke Unit, Affiliated Hospital of Beihua University, Jilin, PR China \\ ${ }^{6}$ Affiliated Hospital of Beihua University, Jilin, PR China
}

\begin{abstract}
Background: Advanced glycation end products (AGEs) are reported to be associated with diabetic vascular complications. However, the role of AGEs in the pathogenesis of cerebral thrombosis remains unclear. This study aimed to investigate the relationship between advanced glycation end products (AGEs) generation and pathogenesis of cerebral thrombosis.

Methods: 100 patients with cerebral thrombosis were recruited and $\mathbf{5 0}$ healthy controlled individuals were served as a control group. Enzyme Linked Immunosorbent Assay (ELISA) was applied for measuring serum AGEs level. The condition of carotid intima was examined by carotid ultrasonography. Serum AGEs levels were compared among patients with certain conditions of carotid atherosclerosis.

Results: AGEs levels in carotid plaque or thickening groups showed significant difference compared with those in control group. Carotid plaque patients showed remarkably different AGEs levels compared to thickening patients. AGEs levels were significantly elevated with higher severity of carotid plaque. Moderate or severe stenosis patients, or blocked group showed significantly different AGEs compared to mild stenosis group. Severe stenosis or blockade group showed remarkably different AGEs compared with moderate stenosis patients. In addition, severe stenosis group had statistically different AGEs compared with blockade group.

Conclusion: AGEs level is correlated with pathogenesis of cerebral thrombosis and they are further elevated with aggravation of carotid atherosclerosis in patients, suggesting they might be used as a biomarker to evaluate the severity of atherosclerosis.
\end{abstract}

Keywords: Advanced glycation end products, Pathogenesis of cerebral thrombosis, Carotid atherosclerosis.

\section{Introduction}

Cerebral thrombosis severely threatens public health, and it is critical to investigate its risk factors for further prevention and treatment [1-4]. Advanced Glycation End Products (AGEs) are heterogeneous, long-lived protein adducts which are generated from a nonenzymatic chemical reaction between sugars and the amino acid [5]. The formation and accumulation of AGEs have been demonstrated to progress during normal aging process as well as at an accelerated rate under diabetes [6]. Circulating levels of AGEs, also known as hemoglobin A1C, might be used as important biomarkers in clinic for monitoring diabetes therapy. Under pathological conditions, cellular interactions with AGEs induce biologic responses that have been linked directly to the development of diabetic vascular complications. Via interacting with AGEs (RAGE) receptor, AGEs are demonstrated to be involved in the generation of oxidative stress in various types of cells and subsequently triggering vascular inflammation $[7,8]$, promoting the proliferation of vascular smooth muscle cells $[9,10]$, potentiating adhesion, aggregation and activation of leukocyte [11], accelerating platelet aggregation and fibronectin formation, elevating macrophage infiltration [12], leading to the occurrence of lipid metabolic disorder, thus facilitating atherosclerosis [13,14]. However, the exact role of AGEs in the pathogenesis of cerebral thrombosis remains poorly understood. This study 
aimed to investigate the relationship between serum AGEs levels and carotid Inner-Middle Membrane Thickness (IMT) as well as the incidence of carotid cavity stenosis in cerebral thrombosis patients.

\section{Patients and Methods}

\section{Research subject}

A total of 100 cerebral thrombosis patients admitted between November 2012 and November 2013 were recruited. Meanwhile, 50 healthy individuals from normal body examination were served as a control group. Inclusive criteria: Admitted in our department, following the diagnostic criteria of cerebral thrombosis stipulated by $4^{\text {th }}$ Cerebral Vascular Disease Symposium of China. Healthy control group was recruited from out-patient individuals receiving normal body examination. Exclusive criteria: Individuals having acute disease of liver, heart, kidney, and gastrointestinal disease, with recent history of surgery, trauma, infection, malignant tumor, diabetic ketosis, or connective tissue diseases. Those recently taken drugs affecting glucose metabolism or oxidative stress functions were also excluded. This study has been approved by the Ethnic Committee of the Affiliated Hospital of Beihua University, Jilin, China and all individuals signed the informed consents before enrolment.

\section{Collection of clinical information}

100 cerebral thrombosis patients were recruited, including 50 cases with single cerebral thrombosis (27 males and 23 females, aged between 40 and $78 \mathrm{y}$, average age $=54 \mathrm{y})$ and 50 cases with cerebral thrombosis with diabetes ( 31 males and 19 females, aged between 40 and $78 \mathrm{y}$, average age $=54 \mathrm{y}) .50$ healthy controlled individuals ( 24 males and 26 females, aging between 40 and $71 \mathrm{y}$, average age $=53.5 \mathrm{y}$ ) were also recruited. All individuals were further divided into pure cerebral thrombosis, cerebral thrombosis with diabetes and healthy controlled groups $(\mathrm{N}=50$ each). All patients were further divided into normal, thickening and plaque sub-groups based on Inner-Middle Membrane Thickness (IMT) acquired from carotid ultrasonography. Grouping criteria were: (1) Normal group (IMT $<1.0 \mathrm{~mm}) ; \quad$ (2) Thickening group $(1.0$ $\mathrm{mm}<\mathrm{IMT}<1.5 \mathrm{~mm}$ ); (3) Plaque group (IMT $>1.5 \mathrm{~mm}$ ). Based on stenosis condition of focal carotid cavity, all plaque tissues were further divided into group groups: (1) Mild stenosis, with less than $50 \%$ of stenosis rate in focal cavity caused by the plaque; (2) Moderate stenosis, with 50\% 69\% stenosis rate in plaque-induced lesion; (3) Severe stenosis group, with $70 \% \sim 99 \%$ stenosis rate caused by focal plaque in carotid cavity; (4) Complete blockade group. All these groups showed no significant difference of sex ratio or age distribution $(\mathrm{p}>0.05)$.

\section{Sample collection and assays}

$4 \mathrm{ml}$ blood samples were collected from elbow veins of patients after $12 \mathrm{~h}$ fasting. Blood samples were incubated for $20 \mathrm{~min}$ at room temperature, and centrifuged for $20 \mathrm{~min}$ (2000 3000 rpm) at room temperature. $200 \mu \mathrm{L}$ serum samples were aliquoted and stored at $-20^{\circ} \mathrm{C}$. ELISA was applied to measure serum AGEs level using test kit (Dongge Boye Biotech, China) following the manual protocols.

\section{Carotid ultrasonography examination}

ACUSON X300 ultrasound apparatus (Siemens, Germany) with VF10-5 and CH5-2 probes was used for carotid ultrasonography examination by clinicians from department of neurology. Patients were placed in supine position with neck extension and head inclined toward the side of examiner. Ultrasonography was performed on bilateral common carotid, internal/external carotid, spinal artery, subclavian artery to examine vascular morphology, structure and plaque formation. IMT was measured simultaneously at $1.0 \sim 1.5 \mathrm{~cm}$ inferior of the branching point of internal and external carotid artery on the distal site of common carotid artery.

\section{Blood pressure measurement}

All individuals were prohibited from smoking within $30 \mathrm{~min}$ before testing. After $10 \mathrm{~min}$ general break, patients were placed in supine or sitting position, with right elbow at identical level of the heart. Table sphygmomanometer was used to measure systolic and diabolic pressure of patients. Blood pressures were measured in three times on different days. All measurements were averaged for calculating Pulse Pressure (PP).

\section{Biochemical index assay}

All individuals were measured for those biochemical indexes by our laboratory: fasted blood Glucose (GLU), Triglyceride (TG), Total Cholesterol (CHO), Low Density Lipoprotein (LDC-C) and High Density Lipoprotein (HDL-C).

\section{Statistical analysis}

SPSS 18.0 software was used to process all data, which were presented as mean \pm Standard Deviation (SD). Measurement data were compared and analysed by one-way Analysis of Variance (ANOVA) with Newman-Keuls multiple comparison post-hoc analysis. A statistical analysis was defined when $\mathrm{p}<0.05$. All statistical analysis was performed using SPSS 18.0 software.

\section{Results}

\section{Comparison of clinical data and AGEs levels}

No statistical significance was found regarding the HDL-C levels in cerebral thrombosis group and control group 
$(\mathrm{p}>0.05)$. However, PP, CHO, LDL-C and AGEs were all significantly higher in cerebral thrombosis than those in control group $(\mathrm{p}<0.01)$. AGEs and PP also showed statistically significant difference among these two groups $(\mathrm{p}<0.01$, Table $1)$.

Table 1. Comparison of clinical information and AGEs.

\begin{tabular}{|c|c|c|c|c|c|c|c|}
\hline \multirow[t]{2}{*}{ Group } & \multirow[t]{2}{*}{$\mathbf{N}$} & PP & $\mathrm{CHO}$ & TG & LDL-C & HDL-C & AGEs \\
\hline & & $\mathrm{mmHg}$ & $\mathrm{mmol} / \mathrm{L}$ & $\mathrm{mmol} / \mathrm{L}$ & $\mathrm{mmol} / \mathrm{L}$ & $\mathrm{mmol} / \mathrm{L}$ & $\mathrm{pg} / \mathrm{ml}$ \\
\hline Control group & 50 & $53.0 \pm 10.7$ & $4.8 \pm 0.7$ & $1.2 \pm 0.5$ & $2.5 \pm 0.4$ & $1.1 \pm 0.4$ & $30.4 \pm 6.9$ \\
\hline Pure cerebral thrombosis & 50 & $62.3 \pm 13.5^{*}$ & $5.5 \pm 0.7^{*}$ & $1.6 \pm 0.7^{*}$ & $2.7 \pm 0.6^{*}$ & $1.1 \pm 0.3$ & $34.2 \pm 7.8^{*}$ \\
\hline Cerebral thrombosis with diabetes & 50 & $73.4 \pm 14.5^{*} @$ & $5.5 \pm 0.8^{*}$ & $1.8 \pm 0.6^{*}$ & $2.8 \pm 0.7^{*} @$ & $1.1 \pm 0.3$ & $38.4 \pm 7.8^{*} @$ \\
\hline
\end{tabular}

" $p<0.01$ compared to control group; $@_{p}<0.01$ compared to pure cerebral thrombosis group.

\section{Comparison of AGEs and PP among different IMT patients}

Compared with control group, plaque and thickening group all showed statistically significant difference of AGEs and PP levels. Comparison between plaque and thickening groups also showed statistically significant difference of AGEs and PP levels (Table 2).

Table 2. Comparison of AGEs and PP levels among IMT patients.

\begin{tabular}{llll}
\hline IMT condition & N & AGEs & PP \\
\cline { 3 - 4 } & & $(\boldsymbol{\mu g} / \mathbf{m l})$ & $\mathbf{( m m H g )}$ \\
\hline Control group & 35 & $25.6 \pm 2.8$ & $61.3 \pm 14.2$ \\
\hline Thickening group & 65 & $36.4 \pm 6.8^{*}$ & $70.5 \pm 12.3^{*}$ \\
\hline Plaque group & 50 & $43.0 \pm 4.5^{* *} @$ & $80.2 \pm 13.0^{*} @$
\end{tabular}

${ }^{*} p<0.05$ and ${ }^{* *} p<0.01$ comparing to control group; $@_{p}<0.05$ and $@ @_{p}<0.01$ comparing to thickening group.

\section{Comparison of AGEs and PP}

AGEs level was elevated along with aggravation of carotid plaque. In moderate and severe stenosis groups, or complete blockade group, both AGEs and PP levels showed statistically significance compared to those in mild stenosis group. Severe stenosis and blockade groups further had remarkably different AGEs and PP levels against those in moderate stenosis group. Moreover, severe stenosis group showed statistically significant difference of AGEs or PP levels compared to complete blockade group (Table 3).

Table 3. Comparison of AGEs and PP among patients with different stenosis conditions.

\begin{tabular}{llll}
\hline Degree of stenosis & $\mathbf{N}$ & AGEs & PP \\
\cline { 3 - 4 } & & $(\mu \mathrm{g} / \mathrm{ml})$ & $(\mathbf{m m H g})$ \\
\hline Mild stenosis & 15 & $32.3 \pm 5.7$ & $62.3 \pm 13.5$ \\
\hline Moderate stenosis & 15 & $35.9 \pm 6.8^{*}$ & $68.5 \pm 11.3^{*}$ \\
\hline Severe stenosis & 12 & $39.3 \pm 5.8^{* *} @$ & $74.9 \pm 12.0^{*} @$ \\
\hline
\end{tabular}

Complete blockade $8 \quad 43.4 \pm 8.1^{* * @ @ \# ~} \quad 80.5 \pm 7.3^{*} @ @ \#$

* $p<0.05$ and ${ }^{* *} p<0.01$ comparing to mild stenosis group; $@_{p}<0.05$ and $@ @ p<0.01$ comparing to moderate stenosis group; ${ }^{*} p<0.05$ comparing to severe stenosis group.

\section{Discussion}

Carotid Atherosclerosis (AS) is common disorder in elder patients. Mild AS usually does not affect brain blood supply. Under severe conditions, however, AS may lead to restricted blood supply toward the brain, leading to symptoms including headache and dazedness. Further activity of neck may further aggravate blood ischemia, or even lead to the formation of cerebral thrombosis $[15,16]$. AS is the basic pathogenic factor for cerebral thrombosis, and is caused by deposition of lipid components of blood onto artery endothelial layer under various stimuli. Such lipid deposition may cause fibrous thickened lesion of endothelial tissues, leading to necrosis and breakdown of deeper tissues, thus causing the formation of plaque-like lesion, and subsequent rigidity of artery walls. Various factors including hypertension, high blood lipid and diabetes all can accelerate AS progression [17-19]. Due to blood flow restriction inside artery with thrombosis, innervated cerebral tissues may not obtain sufficient blood or oxygen supply, leading to tissue softening and necrosis. Clinical manifestations include symptoms and body signs of focal cerebral dysfunction, such as cognitive disorder, sensory dysfunction, paralysis, or aphasia. The observation of carotid artery AS plaque is an important indicator for preventing the development of cerebral thrombosis [20-22].

Currently many reports showed the important role of AGEs in the pathogenesis of AS [21-23]. Related functions mainly include the following contents: Firstly, AGEs may lead to instability and secondary rupture of plaque surface fibrous layer. Previous study showed 3-5 folds higher activity of MMP-9 protein in the shoulder area of unstable plaque compared to those in stable plaques. AGE can facilitate AS pathogenesis progression via high glucose-induced expression of lipoprotein esterase from macrophage, and finally cause the rupture of AS plaque on diabetic patients through facilitating the secretion of inflammatory cytokines such as TNF-A and interleukin-1B from monocytes. Secondly, AGEs can facilitate 
the generation of oxidative stress, which further accelerates AS. Recent in vitro studies [24-26] also confirmed that AGE could stimulate oxidative oxygen production within circulation and vascular cells, and reactive oxygen level is an important factor catalysing AGE generation. Inside AS plaque, upregulation of AGE receptor expression inside monocytes facilitates cellular binding to more AGEs to stimulate the release of pro-inflammatory factors, forming a positive feedback loop of inflammation. Such process can further aggravate pro-inflammatory effects of AGE at local circulation or deposition, thus aggravating the instability of AS plaque. In a word, AGEs showed a wide spectrum of pathogenic roles.

Our results showed significantly higher serum AGEs levels in carotid AS patients, especially those with carotid artery plaque, than those without AS or plaque. Further comparison among different grades of cavity stenosis showed aggravated AGEs displayed higher severity of carotid AS, indicating the association of serum AGEs level with carotid AS or plaque formation. Meanwhile, sub-grouping of patients based on disease conditions showed higher AGEs levels in cerebral thrombosis patients with diabetes compared to those with pure cerebral thrombosis formation or healthy individuals, and those pure thrombosis patients had higher AGEs levels than control group, indicating important roles of AGEs in the formation of cerebral thrombosis. Meanwhile, we also showed that individuals having carotid AS, especially with carotid artery plaque, had significantly higher PP levels than those without carotid AS or plaque. Disease-based grouping showed higher PP levels in cerebral thrombosis patients complicated with diabetes compared to those having pure cerebral thrombosis, which further showed higher PP levels than control group. These results showed the involvement of PP in carotid AS, plaque or cerebral thrombosis formation. PP level is an important indicator reflecting major artery AS, as elevated PP level could accelerate AS progression, and aggravate carotid artery IMT. This study also found better blood pressure management in IMT thickening and control groups compared to plaque group, indicating important roles of PP in AS pathogenesis. In this study, some patients were complicated with diabetes and no significant difference was found in fasted blood glucose level among all groups, probably due to blood glucose management by insulin or oral medicines. However, due to limited number of patients enrolled in this study, large cohort clinical study is required to confirm these findings in the future $[27,28]$.

\section{Conclusion}

AGEs level is correlated with cerebral thrombosis and might work as an indicator for evaluating carotid artery AS condition. Further study on functional roles and mechanism of AGEs in AS probably will provide novel targets for clinical prevention and treatment of AS or cerebral thrombosis. Meanwhile, identification of drugs inhibiting AGEs production or functions also has critical importance for preventing and treatment of cerebral vascular disease.

\section{Acknowledgments}

This work was supported by Jilin Provincial Education Department " $12^{\text {th }}$ Five-Year" science and technology research project, Ji Jiao Ke 2014 (No.498).

\section{References}

1. Silvis SM, Middeldorp S, Zuurbier SM, Cannegieter SC, Coutinho JM. Risk factors for cerebral venous thrombosis. Semin Thromb Hemost 2016; 42: 622-631.

2. Zhang S, Zhao H, Li H, You C, Hui X. Decompressive craniectomy in hemorrhagic cerebral venous thrombosis: clinicoradiological features and risk factors. J Neurosurg 2016; 1-7.

3. Saadatnia M, Fatehi F, Basiri K, Mousavi SA, Mehr GK. Cerebral venous sinus thrombosis risk factors. Int J Stroke 2009; 4: 111-23.

4. de Freitas GR, Bogousslavsky J. Risk factors of cerebral vein and sinus thrombosis. Front Neurol Neurosci 2008; 23: 23-54.

5. Brownlee M, Cerami A, Vlassara H. Advanced glycosylation end products in tissue and the biochemical basis of diabetic complications. N Engl J Med 1988; 318: 1315-21.

6. Yamagishi S. Role of advanced glycation end products (AGEs) and receptor for AGEs (RAGE) in vascular damage in diabetes. Exp Gerontol 2011; 46: 217-224.

7. Zhang H, Tang L, Chen S, Yang Y, Chen M, Luo J. Effect of advanced glycation end products on the expression of hypoxia-inducible factor-1alpha and vascular endothelial growth factor proteins in RF/6A cells. Exp Ther Med 2013; 5: 1519-1522.

8. Zhan Y, Sun HL, Chen H, Zhang H, Sun J, Zhang Z, Cai DH. Glucagon-like peptide-1 (GLP-1) protects vascular endothelial cells against advanced glycation end products (AGEs)-induced apoptosis. Med Sci Monit 2012; 18: 286-291.

9. Nam MH, Son WR, Lee YS, Lee KW. Glycolaldehydederived advanced glycation end products (glycol-AGEs)induced vascular smooth muscle cell dysfunction is regulated by the AGES-receptor (RAGE) axis in endothelium. Cell Commun Adhes 2015; 22: 67-78.

10. Simard E, Sollradl T, Maltais JS, Boucher J, DOrleansJuste P, Grandbois M. Receptor for advanced glycation end-products signaling interferes with the vascular smooth muscle cell contractile phenotype and function. PLoS One 2015; 10: 0128881.

11. Baye E, de Courten MP, Walker K, Ranasinha S, Earnest A, Forbes JM, de Courten B. Effect of dietary advanced glycation end products on inflammation and cardiovascular risks in healthy overweight adults: a randomised crossover trial. Sci Rep 2017; 7: 4123.

12. Byun K, Yoo Y, Son M, Lee J, Jeong GB, Park YM, Salekdeh GH, Lee B. Advanced glycation end-products produced systemically and by macrophages: a common 
contributor to inflammation and degenerative diseases. Pharmacol Ther 2017; 177: 44-55.

13. Xu Z, Liu X, Wang Z, Tao J, Han Z, Gu M, Zhang W, Tan R. Effect of sirolimus on arteriosclerosis induced by advanced glycation end products via inhibition of the ILK/ mTOR pathway in kidney transplantation recipients. Eur J Pharmacol 2017; 17: 30445-30454.

14. Liu X, Liu K, Wang Z, Liu C, Han Z, Tao J, Lu P, Wang J, Wu B, Huang Z, Yin C, Gu M, Tan R. Advanced glycation end products accelerate arteriosclerosis after renal transplantation through the AGE/RAGE/ILK pathway. Exp Mol Pathol 2015; 99: 312-319.

15. Arkuszewski M, Targosz-Gajniak M, Swiat M, Baron J, Zbroszczyk M, Jaworski M, Pieta M, Gruszczynska K, Opala G. Acute intracranial in-stent thrombosis after angioplasty of middle cerebral artery symptomatic stenosis: a case report. Neurologist 2012; 18: 290-295.

16. Tomonori T, Keiko S, Shinkichi H, Yoji N, Akira T. Carotid atherosclerosis and arterial peripheral pulse wave velocity in cerebral thrombosis. J Clin Neurosci 2006; 13: 45-49.

17. Hinduja A, Gupta H, Dye D. Autopsy proven causes of in hospital mortality in acute stroke. J Forensic Leg Med 2013; 20: 1014-1017.

18. Derdeyn CP, Fiorella D, Lynn MJ, Rumboldt Z, Cloft HJ, Gibson D, Turan TN, Lane BF, Janis LS, Chimowitz MI. Mechanisms of stroke after intracranial angioplasty and stenting in the SAMMPRIS trial. Neurosurgery 2013; 72: 777-795.

19. Endo H, Nakagawara J, Okamoto N, Osato T, Kamiyama K, Nakamura H. Development of transdural anastomosis after craniotomy in a patient with atherothrombotic carotid occlusion. J Stroke Cerebrovasc Dis 2012; 21: 911.e1-3.

20. Ichord R, Cerebral Sinovenous Thrombosis. Front Pediatr 2017; 5: 163.

21. Silvis SM, de Sousa DA, Ferro JM, Coutinho JM. Cerebral venous thrombosis. Nat Rev Neurol 2017; 13: 555-565.

22. Hiltunen S, Putaala J, Haapaniemi E, Tatlisumak T. Longterm outcome after cerebral venous thrombosis: analysis of functional and vocational outcome, residual symptoms, and adverse events in 161 patients. J Neurol 2016; 263: 477-484.

23. Vazzana N, Guagnano MT, Cuccurullo C, Ferrante E, Lattanzio S, Liani R, Romano M, Davi G. Endogenous secretory RAGE in obese women: association with platelet activation and oxidative stress. J Clin Endocrinol Metab 2012; 97: 1726-1730.

24. Yan SF, Ramasamy R, Schmidt AM. The RAGE axis: a fundamental mechanism signaling danger to the vulnerable vasculature. Circ Res 2010; 106: 842-853.

25. Barlovic DP, Thomas MC, Jandeleit-Dahm K. Cardiovascular disease: whats all the AGE/RAGE about? Cardiovasc Hematol Disord Drug Targets 2010; 10: 7-15.

26. Yan SF, Ramasamy R, Schmidt AM. Soluble RAGE: therapy and biomarker in unraveling the RAGE axis in chronic disease and aging. Biochem Pharmacol 2010; 79: 1379-1386.

27. Harja E, Bu DX, Hudson BI, Chang JS, Shen X, Hallam K, Kalea AZ, Lu Y, Rosario RH, Oruganti S, Nikolla Z, Belov D, Lalla E, Ramasamy R, Yan SF, Schmidt AM. Vascular and inflammatory stresses mediate atherosclerosis via RAGE and its ligands in apoE-/- mice. J Clin Invest 2008; 118: 183-194.

28. Berrou J, Tostivint I, Verrecchia F, Berthier C, Boulanger E, Mauviel A, Marti HP, Wautier MP, Wautier JL, Rondeau E, Hertig A. Advanced glycation end products regulate extracellular matrix protein and protease expression by human glomerular mesangial cells. Int J Mol Med 2009; 23: $513-520$.

\section{*Correspondence to}

Qingshi Zhao

Stroke Ward

Affiliated Hospital of Beihua University

PR China 